Radiologe 2018 $\cdot 58: 906-913$

https://doi.org/10.1007/s00117-018-0459-4

(c) Der/die Autor(en) 2018

CrossMark

\section{A. H. Schuster $\cdot$ N. Reimann}

Lehrabteilung der Universität Innsbruck, Abteilung Radiologie, University of Innsbruck and Medical University of Innsbruck, Landeskrankenhaus Bregenz, Akademisches Lehrkrankenhaus, Bregenz, Österreich

\title{
Biopsien von Nierenläsionen: wann und wie?
}

\section{Einführung}

Durch den vermehrten Einsatz bildgebender Methoden hat die Häufigkeit zufällig entdeckter Nierenläsionen zugenommen [26]. Mittels Ultraschall (US), Computertomographie (CT) und Magnetresonanztomographie (MRT) können die meisten dieser Läsionen akkurat charakterisiert werden. Für zystische Nierenläsionen hilft die Bosniak-Klassifikation bei der Risikoeinschätzung, nichtsdestotrotz bleibt die Diagnostik komplizierter Nierenzysten häufig ein Problem [1] .

Kleine, unter $3 \mathrm{~cm}$ große, nicht eindeutig klassifizierbare Nierenläsionen werden oftmals kontrolliert. Kann eine fokale Nierenläsion nicht als Zyste, Angiomyolipom (AML) oder Onkozytom eingestuft werden, erfolgt die Resektion. In vermehrtem Maße ist der Radiologe heute mit der Anforderung konfrontiert, bei unklaren Nierenläsionen eine Nierenbiopsie zur sicheren Differenzierung zwischen Benignität und Malignität durchzuführen. Dies hat nicht nur mit der steigenden Inzidenz von Nierenzellkarzinomen („renal cell carcinoma“, RCC) und benignen Nierentumoren zu tun, sondern auch seine Ursache in der sich immer mehr durchsetzenden Erkenntnis, dass eine kontrastmittelaufnehmende Weichteilraumforderung der Niere nicht automatisch als RCC eingestuft und operiert werden sollte [17]. Mehrere Studien konnten zeigen, dass $8-27 \%$ aller resezierten soliden Nierentumoren benigne waren und damit unnötig operiert wurden $[13,21]$. Auch der vermehrte Einsatz ablativer Verfahren als Alternative zu invasiven operativen Verfahren bedingt die
Notwendigkeit einer histologisch-bioptischen Sicherung. Durch bessere Biopsietechniken und neue immunhistochemische Analysemöglichkeiten hat sich die bildgesteuerte Nierenbiopsie zu einer sicheren und genauen Methode in der Diagnostik unklarer Nierenläsionen entwickelt [23].

\section{Indikation}

Die Indikation ist im Hinblick auf die technische Durchführbarkeit, Patientenfaktoren und die klinische Relevanz zu prüfen. Vor Biopsie sollten alle bildgebenden Methoden, insbesondere die MRT, ausgeschöpft worden sein und diese ggf. nachgeholt werden. Im Gespräch mit dem zuweisenden Arzt ist die Relevanz einer Nierenbiopsie für das klinische Management zu erörtern. Bei eindeutigen Zeichen einer Malignität und Operabilität ist es sinnvoller, den Patienten direkt einem resezierenden Verfahren oder einer Ablation zuzuführen, da eine Biopsie nur eine Verzögerung bedeuten würde. Auch bei klaren Charakteristika für Benignität ist eine Biopsie abzulehnen, da bei Vorhandensein mancher Kriterien die Diagnose eines benignen Befunds bereits bildgebend mit hoher Genauigkeit gestellt werden kann [1, 12]. Bei folgenden Indikationen kann die bioptische Sicherung einer Nierenläsion indiziert sein [17, 22]:

\section{Unklare Nierenläsion bei extrarenalem} Primum. Die Niere ist das fünfthäufigste Metastasierungsorgan. Bei extrarenalem Primum sind Nierenraumforderungen trotzdem zumeist nicht Metastasen, sondern RCC [18]. Da bei Metastasierung meist eine systemische Therapie durchgeführt wird und im Gegensatz dazu RCC operiert oder abladiert werden, hat das Ergebnis einer Biopsie entscheidenden Einfluss auf die Therapie und Prognose des Patienten.

Inoperables Nierenzellkarzinom. Neue Therapieverfahren erfordern eine Subtypisierung und molekularpathologische Tests, um Vorhersagen über Prognose und Therapieansprechen einer Immuntherapie mit Zytokinen (Interferon alpha, Interleukin 2), monoklonalen Antikörpern (Bevacizumab, Sorafenib etc.), Tyrosinkinaseinhibitoren (Sorafenib, Sunitinib etc.) oder mTOR(,,mechanistic target of rapamycin")-Inhibitoren zu treffen [17].

\section{Nierenzellkarzinomverdacht bei er-} höhter Komorbidität. Bei erhöhter Komorbidität kann die histologische Sicherung eines RCC die Risiko-NutzenAbwägung beeinflussen und das Risiko der unnötigen Operation einer benignen Läsion durch die gering invasivere Biopsie ausgeschlossen werden [14].

\section{Hyperdense kontrastanreichernde Nie-} renläsion $<3 \mathrm{~cm}$. Computertomographisch nicht kontrastanreichernde, hyperdense Nierenläsionen können als eingeblutete Zysten eingestuft und verlaufskontrolliert werden. Bei Konstrastmittelaufnahme bereiten hyperdense Nierenläsionen jedoch diagnostische Probleme. Fettarme AML, Onkozytome und das metanephrische Adenom können bildgebend nur schwer vom RCC unterschieden werden - hier sollte ergänzend zur CT eine MRT durchgeführt 

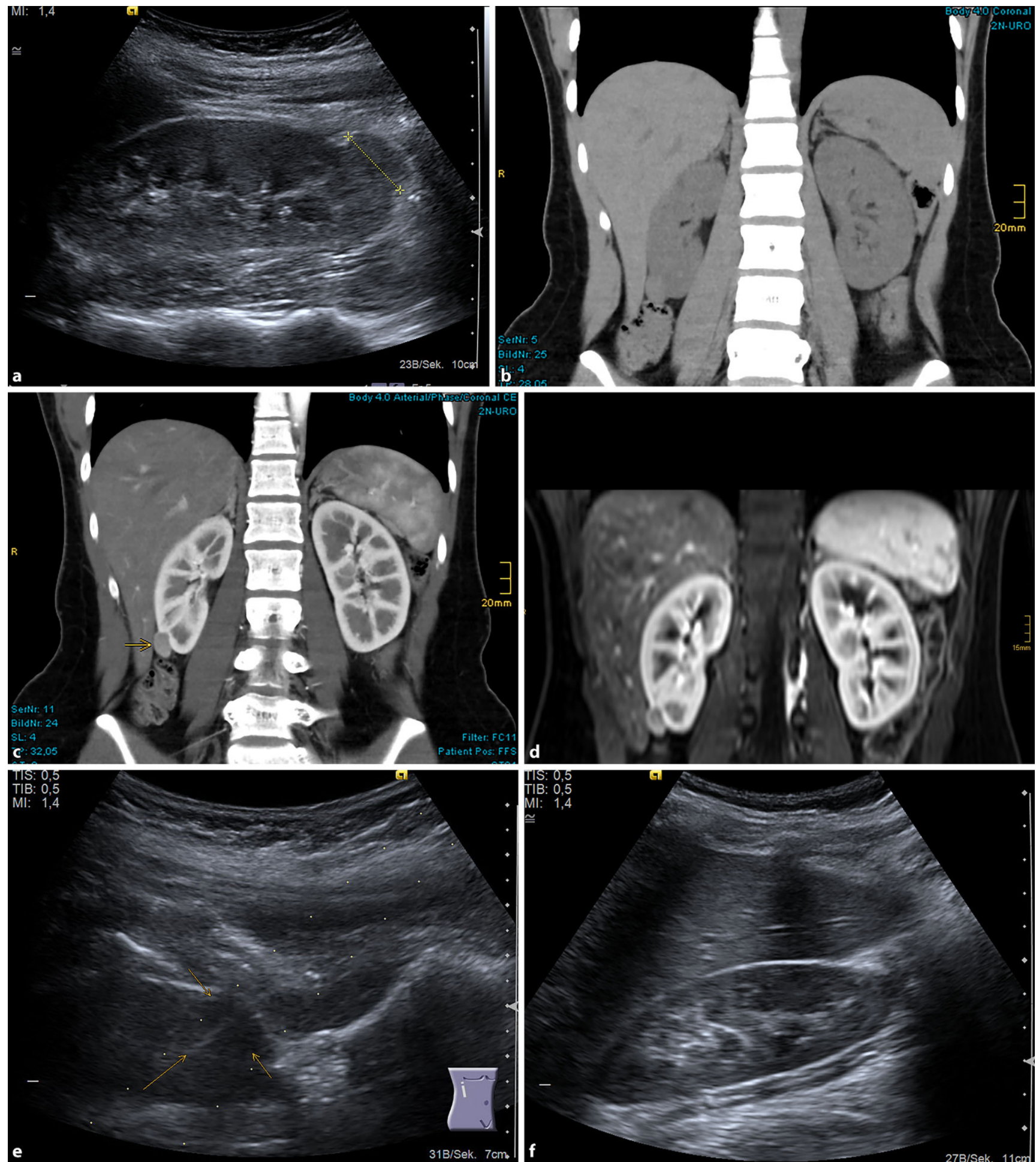

Abb. 1 ॥ Kleine solide Nierenläsion $<3 \mathrm{~cm}$. a Sonographie mit leicht hyperechogenem, $15 \mathrm{~mm}$ großem Tumor am Nierenunterpol.b Koronare CTMPR nativ mit kleiner leicht hyperdenser Nierenläsion am rechten Nierenunterpol.c Die koronare CTMPR in arterieller Phase zeigt eine Kontrastmittelaufnahme der Nierenläsion. $d$ Die koronare 3D-T1-Dixon in arterieller Phase zeigt den hypokontrastierten Knoten. e US-Bild im Flankenschnitt mit abgeschossener Koaxialnadel (langer Pfeil) und leicht hyperechogenem Tumor (kurze Pfeile). f US-Bild im Flankenschnitt am 1. Tag post punktionem, kein Hämatom. Der histologische Befund ergibt ein Onkozytom 
werden. Typische MR-Zeichen eines klarzelligen RCC sind T2-Hyperintensität, mikroskopisches Fett und Hypervaskularisation. Das papilläre RCC ist typischerweise T2-hypointens, hypovaskulär und kann Hämosiderin aufweisen. Das lipidarme AML weist eine T2Hypointensität und Kontrastmittelanreicherung auf. Bei $5 \%$ aller AML kann bildgebend kein Fett nachgewiesen werden [9]. Das chromophobe RCC und das Onkozytom haben ähnliche Histologien und Bildgebungsmerkmale, und auch das klarzellige RCC ist oft nur schwer vom Onkozytom oder fettarmen AML zu unterscheiden $[7,12]$. Kann auch mit multiparametrischer MR-Bildgebung nicht die Diagnose eines AML oder eines klarzelligen bzw. papillären RCC gestellt werden, ist die Nierenbiopsie oder die Kontrolle indiziert [12].

Geplante perkutane Ablation. Die perkutane Ablation hat sich als parenchymschonende Alternative v. a. für Hochrisikopatienten etabliert [6]. Mangels Operationspräparat ist die Intervention durch eine histologische Sicherung mittels Biopsie zu rechtfertigen.

Kleine solide Nierenläsion $(<3 \mathrm{~cm})$ (• Abb. 1). Mit abnehmender Tumorgröße steigt die Wahrscheinlichkeit einer benignen Nierenläsion. Bezüglich der biologischen Aggressivität kleiner RCC gibt es unterschiedliche Ergebnisse; die Erfahrung zeigt aber, dass viele RCC nur langsam wachsen, klinisch stumm verlaufen und bei Vorliegen von Komorbiditäten nicht lebenslimitierend sind. Eine bioptische Sicherung kann hier z. B. durch Bestimmung der Zellsubtypen oder des Graduierungssystems nach Fuhrmann helfen, die Tumoraggressivität und Prognose abzuschätzen [17]. Das Alter und die Lebenserwartung des Patienten, seine Operabilität und Komorbidität sowie der Patientenwunsch sind bei der Wahl des besten Prozedere $\mathrm{zu}$ berücksichtigen.

Einzelniere. Aufgrund des drohenden Parenchymverlusts und einer folgenschweren Dialyse kann die Operationsindikation durch das Ergebnis einer Nierenbiopsie erhärtet werden.

Radiologe 2018 · 58:906-913 https://doi.org/10.1007/s00117-018-0459-4

(c) Der/die Autor(en) 2018

\section{A. H. Schuster · N. Reimann}

\section{Biopsien von Nierenläsionen: wann und wie?}

\section{Zusammenfassung}

Die Nachfrage für bildgesteuerte Nierenbiopsien hat durch die erhöhte Detektion von Nierenläsionen zugenommen. Trotz der modernen Bildgebung können viele kleine Nierentumoren jedoch nicht als gutartig eingestuft werden, da sie von Nierenzellkarzinomen nicht unterscheidbar sind. Die Ultraschall- oder CT-gezielte Nierenbiopsie kann als sichere und genaue Methode in der Diagnostik von Nierenläsionen hilfreich bei der Wahl neuer ablativer und medikamentöser Therapieformen sein und unnötige Operationen vermeiden. Dieser Artikel beschreibt die klinischen Situationen, in denen eine bioptische Sicherung sinnvoll ist, und beleuchtet die Faktoren, die bei der Durchführung einer Biopsie beachtet werden sollten.

Schlüsselwörter

Bildgesteuerte Punktion · Niere · Ultraschall . Computertomographie - Magnetresonanztomographie

\section{Biopsies of kidney lesions: when and how?}

\section{Abstract}

The demand for image-guided renal biopsy has increased due to the better detection of renal lesions; however, despite modern imaging techniques many small renal tumors cannot be classified as benign because they cannot be differentiated from renal cell carcinoma. Ultrasound and computed tomography (CT)-guided kidney biopsy is a safe and accurate method in the diagnostics of renal lesions and can be helpful in the selection of new ablative and pharmaceutical forms of treatment and avoid unnecessary operations. This article describes the clinical indications for an image-guided biopsy and discusses factors which should be considered when performing a biopsy.

Keywords Image-guided biopsy - Kidney - Ultrasound . Computed tomography - Magnetic resonance imaging

\section{Verdacht auf Abszess oder Lymphom.} Für das seltene primäre oder häufigere sekundäre Lymphom der Niere ist eine Biopsie zur histologischen Klassifizierung notwendig. Entzündungen oder Abszesse lassen sich bildgebend in der Regel gut von Nierentumoren differenzieren und können kurzfristig kontrolliert werden. Ein negatives Infektlabor in Blut- und Harnproben sowie eine fehlende bildgebende Rückbildung können aber zur Missinterpretation führen, weshalb eine unnötige chirurgische Resektion durch bioptische Sicherung vermieden werden kann.

Multiple Nierenläsionen. Die hereditäre Onkozytose manifestiert sich durch multiple Onkozytome, die nicht operiert werden müssen. Die Nierenbiopsie ist hier essenziell in der Diagnosefindung.
Komplizierte Nierenzyste Bosniak III. Mithilfe der seit Langem etablierten Bosniak-Klassifikation kann das Malignitätsrisiko zystischer Nierenläsionen genau klassifiziert werden, eine aktuelle Metaanalyse zeigte jedoch eine mäßige Spezifität auf (71\%; [19]). Die Malignitätsraten von Bosniak-IIF- und -II-Zysten sind niedrig, aber nicht irrelevant, weshalb diese bildgebend kontrolliert werden sollten [19]. Entsprechend der Bosniak-Klassifikation sollten Bosniak-III-Zysten laparoskopisch abgeklärt werden. Der Einsatz der Nierenbiopsie könnte hier - v.a. bei erhöhtem Operationsrisiko - eine Alternative sein. In einer Studie mit 28 Patienten konnte bei $39 \%$ der Patienten durch die bildgesteuerte Probenentnahme eine unnötige Operation vermieden werden [3]. 


\section{Planung und Vorbereitung}

Ist einmal die Indikation zur bildgesteuerten Nierenbiopsie gestellt, sollte diese entsprechend den Richtlinien der Cardiovascular and Interventional Radiological Society of Europe (CIRSE) geplant und durchgeführt werden [24]: Labortechnisch sind der INR(International Normalized Ratio)-Wert, die Thrombozytenzahl, der Quicktest, der Hämatokrit und bei Gabe niedrigfraktionierter Heparine die partielle Thromboplastinzeit (PTT) zu bestimmen [16]. Zur Einschätzung der Nierenfunktion werden Harnstoff, Kreatinin und die glomeruläre Filtrationsrate (GFR) bestimmt. Die Laborwerte sollten nicht älter als 30 Tage sein, der INR-Wert unter 1,5, die PTT unter 1,5, der Quickwert über 50 und die Thrombozytenzahl über 50.000 liegen. Manche Autoren empfehlen eine Blutgruppenbestimmung. Eine Blutdrucksenkung bei Hypertonus wird zwar nicht generell empfohlen, es konnte aber ein erhöhtes Risiko für Blutungskomplikationen bei RR(Blutdruck nach Riva-Rocci)-Werten über 140/90 mm Hg nachgewiesen werden [10].

Das Blutungsrisiko für eine Nierenbiopsie wird von der Society of Interventional Radiology in der Kategorie "hoch“ eingestuft. Eine allenfalls vorhandene Blutverdünnung sollte zeitgerecht abgesetzt und eine vorhandene Koagulopathie ggf. korrigiert werden. Für die in den letzten Jahren eingesetzten neuen oralen Antikoagulanzien (NOAC) gibt es keine Antidote; generell sollten sie 1 bis 2 Tage vor Eingriff pausiert und frühestens $24 \mathrm{~h}$ nach Punktion wieder gegeben werden. Clopidgrel und Acetylsalicylsäure (ASS) sind 5 Tage vor Punktion abzusetzen. Die Gabe niedrigfraktionierter Heparine sollte $24 \mathrm{~h}$ vor Eingriff unterbrochen werden.

Patienten sollten 4-6h vor Eingriff nüchtern sein. Eine prophylaktische Antibiose wird generell nicht empfohlen.

Absolute Kontraindikationen sind eine nicht korrigierbare Koagulopathie, ein fehlendes Patienteneinverständnis und das Fehlen eines sicheren Zugangsweges. Bei eingeschränkter PatientenCompliance ist eine Sedierung oder Allgemeinnarkose zu überlegen. Relevante
Komorbiditäten wie eine hämodynamische oder respiratorische Instabilität sind weitere relative Kontraindikationen.

Eine mündliche und schriftliche Aufklärung mittels standardisiertem Aufklärungsbogen $24 \mathrm{~h}$ vor Eingriff ist obligat und die Einwilligung durch Unterschrift von Arzt und Patient zu dokumentieren.

Die Biopsie einer Nierenläsion kann mittels US, CT oder MRT durchgeführt werden. Entscheidend für die Wahl der bildgebenden Methode sind die Sichtbarkeit der relevanten Nierenpathologie und der umgebenden Anatomie, eine durchgehende Darstellbarkeit des Stichkanals und der Punktionsnadel, die frühzeitige Beurteilung möglicher Komplikationen, die weitgehend unbehinderte Durchführung aller Punktionsmanöver, eine angenehme Patientenlagerung und die Limitierung der Strahlenexposition.

Für die Punktion sollte die bildgebende Methode ausgewählt werden, in der die Läsion ohne Kontrastmittelgabe am besten zu sehen ist und welche den sichersten Zugangsweg bietet. Die USgezielte Biopsie ist die primäre Methode der Wahl. Der US ist gut verfügbar und bietet den Vorteil, die Zielläsion und die Nadel auch während der Atemexkursion in Echtzeit darzustellen. Für den Zugangsweg kann ein beliebiger Winkel gewählt werden. Mittels Dopplersonographie können prominente Gefäße dargestellt und eine Gefäßverletzung so besser vermieden werden. Durch den Einsatz von US-Kontrastmitteln können auch Läsionen dargestellt werden, die in der B-Bild-Sonographie nicht sichtbar sind. Moderne US-Geräte bieten Bildfusion von Kontrastmittelsonographie (CEUS) und CT oder MRT ohne und mit Nadelführung an, womit die Vorteile anderer bildgebender Methoden genutzt werden können. Die Bildfusion verbessert die Darstellbarkeit und Dignitätseinschätzung renaler Läsionen im Vergleich zu Schnittbildmethoden allein und ermöglicht komplexe Angulierungszugänge [2, 4]. Neue Navigationssysteme sind in der Lage, mittels elektromagnetischer und optischer Sensoren die Position der Nadel und der Zielläsion in Echtzeit darzustellen und den Nadeltrakt auch außerhalb der Schichtebene $\mathrm{zu}$ visualisieren.
Hier steht eine Anzeige. (2) Springer 
Kann die Zielläsion sonographisch nicht oder im CT besser dargestellt werden, wird umgehend die CT als Zielmethode überprüft. Die CT wird von vielen Radiologen aufgrund des gewohnteren Handlings präferiert. Die MRT hat sich aufgrund der schlechteren Verfügbarkeit und Komplexität nicht etablieren können und bleibt einzelnen Zentren vorbehalten.

An unserer Institution hat es sich von Vorteil erwiesen, die Patienten zur Planungssonographie vor Terminvergabe einzuberufen. Der Planungs-US soll dazu dienen, die technische Durchführbarkeit der Punktion zu bestätigen, und kann für das Aufklärungsgespräch und die Einschätzung der PatientenCompliance genutzt werden.

Nierenbiopsien können zwar bei ausgewähltem Patientengut ambulant oder tagesstationär durchgeführt werden [23], in der Regel empfiehlt sich jedoch ein 24stündiger stationärer Aufenthalt. In einer großen Studie mit 750 Patienten wurde nachgewiesen, dass mehr als $90 \%$ aller schweren Komplikationen innerhalb dieser Zeit auftreten [25].

\section{Durchführung}

\section{US-gezielte Biopsie}

Vor Beginn der Intervention wird ein venöser Zugang gelegt und Sauerstoff (21/min) über die Nasenbrille verabreicht. Puls- und Sättigungskontrolle erfolgen mittels Pulsoxymetrie.

Der Patient wird in Links- bzw. Rechtsseitenlage oder Bauchlage stabil gelagert, wobei auf eine bequeme und armschonende Lagerung geachtet werden sollte, da bei suboptimaler Lagerung die Patienten-Compliance schmerzbedingt mit zunehmender Interventionszeit abnimmt. Eine stabile und fixierte Lagerung ist v. a. unter Verwendung von Navigationssystemen erforderlich.

Als Schallkopfebene wird die Schichtführung gewählt, in der die Zielläsion auch während der Atemexkursion des Patienten am besten sichtbar ist und welche einen sicheren und kurzen $\mathrm{Zu}$ gangsweg bietet. Typischerweise wird ein 2- bis 6-MHz-Konvexschallkopf verwendet. Manche Hersteller bieten eigene Punktions-Presets an, welche die Sichtbarkeit der Nadel verbessern. Die sagittale Schallkopfebene ist von Vorteil, da während der Atmung die Zielläsion zumeist durchweg sichtbar ist. Im Besonderen ist auf Kolon, Milz und bei interkostalem Zugangsweg auf die Interkostalarterie zu achten. Die Verwendung einer Nadelführung am Schallkopf verbessert die Sichtbarkeit der Nadel und die Trefferquote und erlaubt es auch weniger geübten Punkteuren, tiefe Zielläsionen in einem Punktionsvorgang zu treffen [20].

Aufgrund der Atemverschieblichkeit ist es hilfreich, die Atemmanöver mit dem Patienten vor Interventionsbeginn $\mathrm{zu}$ üben. $\mathrm{Ob}$ der Eingriff in Inspiration, Expiration oder flacher Atmung durchgeführt wird, sollte individuell je nach Atemverschieblichkeit, PatientenCompliance, Größe und Zugänglichkeit der Zielläsion entschieden werden. Zur besseren Reproduzierbarkeit ist es hilfreich, die interventionellen Schritte in Atemstillstand durchzuführen und die Atempausen kurz zu halten, um forcierte Atemexkursionen zu vermeiden.

Die Intervention erfolgt in Lokalanästhesie. Eine Sedoanalgesie mit Midazolam kann zwar den Patientenkomfort verbessern, es sollte jedoch die fehlende Kooperation gerade für Atemmanöver bedacht werden. Nach Durchführung der Hautdesinfektion wird unter sterilen Kautelen (sterile Abdecktücher, sterile Schallkopfabdeckung, steriles Ultraschallgel) die Lokalanästhesie entlang des Nadeltrakts mittels 1- bis $2 \%$ igem Lidocain (10-20 ml) durchgeführt, wobei ein Depot direkt an der Nierenkapsel gesetzt werden sollte. Ein längere als die üblicherweise für die Lokalanästhesie verwendeten 21- bis 24-G-Nadeln kann hier von Vorteil sein.

Durch feine „schüttelnde“ Bewegungen kann die Nadelspitze beim Vorschub besser sichtbar gemacht werden. Verschiedene Hersteller bieten Nadeln mit eigenem Schliff an, welche sonographisch besser sichtbar sind. Eine akzidentelle Luftinjektion ist $\mathrm{zu}$ vermeiden, um die Abgrenzbarkeit der Nadel nicht zu vermindern. Die Koaxialnadel wird in kurzem Atemstillstand an die Zielläsion herangeführt und die Probenentnahme zügig durchgeführt. Bei guter Patientenkooperation kann auch durch Korrektur der Atemposition die Zielläsion in eine bessere Schussposition vor die Nadel gebracht werden.

Es ist zu beachten, dass die Nadel nicht zu lange an der Nierenkapsel verweilt, da sich das Risiko einer Nierenkapselverletzung und damit das Blutungsrisiko durch die Atembewegung erhöht. Idealerweise wird deshalb die Koaxialnadel auch kurzstreckig in der Läsion platziert, um häufigere Passagen der Nierenkapsel zu vermeiden. Manche Biopsiesysteme erlauben auch die Einstellung der Vorschusslänge. Vor Auslösen sollte sichergestellt werden, dass beim Vorschuss der schneidende Anteil das Nierenparenchym zum Hilus hin nicht überschreitet, um relevante Blutungen zu vermeiden. Insbesondere bei Probeentnahme von hilusnahen Läsionen sollte mit besonderer Vorsicht vorgegangen werden. Bei kleinen Läsionen in heikler Position kann bei vielen Systemen der geöffnete Schacht der Stanzbiopsienadel in der Zielläsion platziert und dann die Hülse semiautomatisch ausgelöst werden, womit die akzidentelle Verletzung von Gefäßen besser verhindert werden kann.

Die Probenentnahme wird koaxial durchgeführt und ein 17/18-G-Stanzbiopsiesystem verwendet, da im Vergleich zur Nichtkoaxialmethode ein signifikant geringeres Blutungsrisiko nachgewiesen werden konnte [24]. Regelhaft werden 3 bis 4 Proben entnommen. Die korrekte intraläsionale Lage der abgeschossenen Nadel ist bildgebend zu dokumentieren. Für die Gewebeentnahme sollten zellreiche und nichtnekrotische Tumoranteile ausgewählt werden.

Bei erhöhtem Blutungsrisiko oder starker retrograder Blutung aus der Koaxialnadel kann die Verwendung eines Gelatinezylinders (z.B. Spongostan ${ }^{\mathrm{Tm}}$, Ethicon; Hunter@ Sealing Device, Vascular Solutions; Gelfoam $\odot$, Pfizer, etc.) zur Embolisation des Nadeltrakts in Erwägung gezogen werden, wie er für Lungen- oder Leberbiopsien verwendet wird $[8,23,27]$, indes liegen jedoch keine randomisierten kontrollierten Studien für die Effektivität seines Einsatzes vor.

Nach Rückzug der Koaxialnadel wird die Punktionsstelle für mehrere Minuten 
komprimiert. Die Punktionsstelle wird mittels Pflasterverband versorgt.

Nach dem Eingriff werden die entnommenen Gewebezylinder in einem Probenkäfig versorgt und in Formalin fixiert.

\section{CT-gezielte Biopsie}

Bei der Patientenlagerung ist darauf zu achten, dass zwischen Patient und CTGantry genügend Platz für die Manipulationen während der CT-gezielten Biopsie verbleibt. Um die Atemverschieblichkeit der zu punktierenden Nierenläsion zu verringern, erfolgt die Lagerung in Linksoder Rechtsseitenlage auf der zu punktierenden Seite unten. Je nach Lage und Zugänglichkeit kann auch eine Bauchoder Rückenlage oder eine unterpolsterte Schräglage nach ventral oder dorsal notwendig sein. Alle Maßnahmen der Strahlendosisverminderung sollten ausgeschöpft werden, um die Strahlenbelastung des Patienten und des Interventionsteams gering zu halten, die Dosis jedoch nur derart vermindert werden, dass eine ausreichende Bildqualität garantiert ist. Eine Reduktion der Schichtdicke oder eine Verwendung weicherer Filter kann zu einem verbesserten Signal-Rausch-Verhältnis beitragen.

\section{Nachsorge}

Die meisten Komplikationen treten 4-6h nach Punktion auf, weshalb die Patienten eine 6-stündige Bettruhe und Nüchternheit halten müssen und überwacht werden. Der Blutdruck sollte stündlich kontrolliert und der Patient nach Rückenschmerzen gefragt werden. Insgesamt verbleibt der Patient $24 \mathrm{~h}$ postinterventionell stationär. Klagt ein Patient über zunehmende Rückenschmerzen, ist ein Blutbild zu bestimmen und eine CT-Kontrolle in Erwägung zu ziehen. Vor Entlassung werden Komplikationen durch eine abschließende Bildgebung, vorzugsweise US, ausgeschlossen, und der Patient wird über Verhaltensregeln, Spätkomplikationen und deren Symptome informiert [11].

\section{Genauigkeit}

Sensitivität und Spezifität der Stanzbiopsie betragen 97,5\% bzw. 96,2\%, der PPV(,positive predictive value“)-Wert wird mit 99,8\%, der NPV(,negative predictive value")-Wert mit $68,5 \%$ angegeben. Die Rate nichtdiagnostischer Biopsien wird in einer Metaanalyse mit $14,1 \%$ angegeben, $90,4 \%$ der erfolglos punktierten Tumoren stellten sich im chirurgischen Operationspräparat als maligne heraus [15]. Bei nichtdiagnostischem Ergebnis ist eine Rebiopsie empfehlenswert, da diese meistens erfolgreich ist [15]. Die Feinnadelbiopsie hat eine geringere Sensitivität von $62,5 \%$ und sollte unserer Meinung nach nur in Kliniken mit Zugang zu einer Pathologie mit hoher zytologischer Expertise durchgeführt werden.

\section{Komplikationen}

Die bildgesteuerte Nierenbiopsie ist durch die Verwendung dünnerer $\mathrm{Na}$ deln eine sichere Methode geworden [22]. Das Risiko für Komplikationen wird in der Literatur mit 1,2-6,6\% angegeben und ist bei Patienten mit fortgeschrittener Niereninsuffizienz höher [25]. Starke Schmerzen werden bei 1,2\% der Patienten beschrieben [15].

Die Blutung ist die häufigste Komplikation einer Nierenbiopsie und tritt in Form eines perirenalen oder subkapsulären Hämatoms in unterschiedlicher Ausprägung als „regelhafte“ und punktionsbedingte Komplikation bei bis zu $80 \%$ aller Punktionen auf. Nur bei $6 \%$ der Patienten ist sie klinisch relevant [25]. In der Regel können postpunktionelle Blutungen konservativ beobachtet werden und mittels regelmäßiger Bildgebung und Hämoglobinbestimmung kontrolliert werden. Bluttransfusionen nach Nierenpunktion sind nur in 1,5\% der Fälle notwendig [22].

Eine Mikrohämaturie ist meist nicht signifikant und verschwindet in der Regel innerhalb von $48 \mathrm{~h}$. Eine Makrohämaturie wird in $1 \%$ aller Fälle beobachtet und ist meist selbstlimitierend. Persistiert sie, sollte an eine arteriovenöse Fistel gedacht werden [15].
Das Risiko einer arteriovenösen Fistel nach Nierenpunktion wird mit $0,4 \%$ berichtet und korreliert mit der gewählten Nadelgröße, weshalb die Verwendung von 17/18-G- oder 19/20-G-Sytemen empfohlen wird. $70 \%$ verlaufen asymptomatisch und verschließen sich nach einigen Wochen selbst, $30 \%$ sind klinisch manifest und benötigen Bluttransfusionen. Eine Embolisation ist bei Nachweis eines Pseudoaneurysmas oder bei klinisch relevanten arteriovenösen Fisteln indiziert.

Das Risiko eines Pneumothorax wird mit $0,6 \%$ beschrieben und kann vermindert werden, wenn der Patient auf der Punktionsseite liegt, was v. a. bei der CT-gezielten Punktion genutzt werden kann, bei der US-gezielten Biopsie jedoch häufig von Nachteil ist [15, 23]. Bei V.a. Pneumothorax nach US-gezielter Punktion wird unmittelbar nach Beendigung der Intervention ein Thoraxröntgen stehend angefertigt. Bei Auftreten eines Pneumothorax in der CT kann dieser sofort mittels Venflon ${ }^{\circledR}$ abgezogen werden und ggf. mittels kleiner Pleuradrainage (Pleuracath ${ }^{\circledR}$, etc.) versorgt werden. Ein postpunktioneller Pneumothorax ist häufig selbstlimitierend und benötigt keine weitere Therapie. Üblicherweise muss bei Vorliegen eines Pneumothorax 24h Bettruhe gehalten und nach $4 \mathrm{~h}$ ein Kontrollthoraxröntgen stehend angefertigt werden, um eine Progredienz auszuschließen.

Postpunktionelle Infektionen sind mit $0,2 \%$ sehr selten. Die Mortalität wird mit $0,031 \%$ und das Risiko eines Organverlustes mit $0,1 \%$ berichtet [23].

Das Risiko einer Tumorzellverschleppung entlang des Nadeltrakts ist mit $0,01 \%$ vernachlässigbar gering und damit niedriger als bei anderen primären und sekundären Tumoren [5]. In der Literatur sind nur einzelne Fälle beschrieben. Bei potenziell resektablen Fällen sollten nichtsdestotrotz die Vorteile und Risiken gegeneinander abgewogen werden. Das Risiko scheint bei bildgebend aggressiv infiltrativ wachsenden Tumoren, urothelialen Karzinomen und den seltenen Sarkomen höher zu sein als bei expansiv langsam wachsenden Tumoren. 


\section{Fazit für die Praxis}

- Die bildgesteuerte Biopsie ist eine sichere und genaue Methode in der Diagnostik unklarer Nierenläsionen und gewinnt durch die erhöhte Inzidenz von Nierenläsionen an Bedeutung.

- Indikationen für die US- oder CTgezielte Nierenbiopsie sind Patienten mit Nierenraumforderungen und extrarenalem Primum, nichtresektablem Nierenzellkarzinom, unklaren zystischen Läsionen, einer hyperdensen kontrastanreichernden Nierenläsion und erhöhter Komorbidität.

- Mit Hilfe der gezielten Nierenbiopsie können unnötige Operationen benigner Tumoren vermieden werden und eine Übertherapie langsam wachsender und subklinischer RCC bei selektierten Patienten abgewogen werden.

- Die bildgesteuerte Biopsie sichert die Indikation vor Nierentumorablation und ermöglicht den besseren Einsatz moderner medikamentöser Therapieformen des RCC.

\section{Korrespondenzadresse}

A.H. Schuster
Lehrabteilung der Universität
Innsbruck, Abteilung
Radiologie, University of
Innsbruck and Medical
University of Innsbruck,
Landeskrankenhaus
Bregenz, Akademisches
Lehrkrankenhaus
Carl-Pedenz-Straße 2,
6900 Bregenz, Österreich
antonius.schuster@lkhb.at

Funding. Open access funding provided by University of Innsbruck and Medical University of Innsbruck.

\section{Einhaltung ethischer Richtlinien}

Interessenkonflikt. A.H. Schuster und N. Reimann geben an, dass kein Interessenkonflikt besteht.

Dieser Beitrag beinhaltet keine von den Autoren durchgeführten Studien an Menschen oder Tieren.

Open Access. Dieser Artikel wird unter der Creative Commons Namensnennung 4.0 International Lizenz (http://creativecommons.org/licenses/by/4.0/deed. de) veröffentlicht, welche die Nutzung, Vervielfältigung, Bearbeitung, Verbreitung und Wiedergabe in jeglichem Medium und Format erlaubt, sofern Sie den/die ursprünglichen Autor(en) und die Quelle ordnungsgemäßnennen, einen Link zur Creative Commons Lizenz beifügen und angeben, ob Änderungen vorgenommen wurden.

\section{Literatur}

1. Bosniak MA (2012) The Bosniak renal cyst classification: 25 years later. Radiology 262:781-785

2. Clevert DA, Jung EM (2013) Interventional sonography of the liver and kidneys. Radiologe 53:962-973

3. Harisinghani MG, Maher MM, Gervais DA et al (2003) Incidence of malignancy in complex cystic renal masses (Bosniak category III): Should imaging-guided biopsy precede surgery? Ajr Am J Roentgenol 180:755-758

4. Helck $A$, D'anastasi $M$, Notohamiprodjo $M$ et al (2012) Multimodality imaging using ultrasound image fusion in renal lesions. Clin Hemorheol Microcirc 50:79-89

5. Herts BR (2000) Imaging guided biopsies of rena masses. Curr Opin Urol 10:105-109

6. Higgins LJ, Hong K (2015) Renal ablation techniques: State of the Art. Ajr Am J Roentgenol 205:735-741

7. Hotker AM, Mazaheri Y, Wibmer A et al (2017) Differentiation of clear cell renal cell carcinoma from other renal cortical tumors by use of a quantitative multiparametric MRI approach. Aj Am JRoentgenol 208:W85-W91

8. Kilcoyne A, Gervais DA (2016) Kidney, ureter, and bladder biopsy. Tech Vasc Interv Radiol 19:237-244

9. Kim JK, Park SY, Shon JH et al (2004) Angiomyolipoma with minimal fat: Differentiation from renal cell carcinoma at biphasic helical CT. Radiology 230:677-684

10. Kriegshauser JS, Patel MD, Young SW et al (2015) Risk of bleeding after native renal biopsy as a function of preprocedural systolic and diastolic blood pressure. JVasc Interv Radiol 26:206-212

11. Lee MJ, Fanelli F, Haage P et al (2012) Patient safety in interventional radiology: A CIRSE IR checklist. Cardiovasc Intervent Radiol 35:244-246

12. Lopes Vendrami C, Parada Villavicencio C, Dejulio TJ et al (2017) Differentiation of solid renal tumors with multiparametric MR imaging. Radiographics 37:2026-2042

13. Mckiernan J, Yossepowitch O, Kattan MW et al (2002) Partial nephrectomy for renal cortical tumors: pathologic findings and impact on outcome. Urology 60:1003-1009

14. Niceforo J, Coughlin BF (1993) Diagnosis of renal cell carcinoma: Value of fine-needle aspiration cytology in patients with metastases or contraindications to nephrectomy. Ajr Am J Roentgenol 161:1303-1305

15. Patel HD, Johnson MH, Pierorazio PM et al (2016) Diagnostic accuracy and risks of biopsy in the diagnosis of a renal mass suspicious for localized renal cell carcinoma: Systematic review of the literature. JUrol 195:1340-1347

16. Patel IJ, Davidson JC, Nikolic B et al (2012) Consensus guidelines for periprocedural management of coagulation status and hemostasis risk in percutaneous image-guided interventions. J Vasc Interv Radiol 23:727-736

17. Sahni VA, Silverman SG (2009) Biopsy of renal masses: When and why. Cancer Imaging 9:44-55
18. Sanchez-Ortiz RF, Madsen LT, Bermejo CE et al (2004) A renal mass in the setting of a nonrenal malignancy: When is a renal tumor biopsy appropriate? Cancer 101:2195-2201

19. Sevcenco S, Spick C, Helbich TH et al (2017) Malignancy rates and diagnostic performance of the Bosniak classification for the diagnosis of cystic renal lesions in computed tomography a systematic review and meta-analysis. Eur Radiol 27:2239-2247

20. Shabana W, Kielar A, Vermani V et al (2013) Accuracy of sonographically guided biopsy using a freehand versus needle-guided technique: Computed tomographic correlation study. JUltrasound Med 32:535-540

21. Silver DA, Morash C, Brenner P et al (1997) Pathologic findings at the time of nephrectomy for renal mass. Ann Surg Oncol 4:570-574

22. Silverman SG, Gan YU, Mortele KJ et al (2006) Renal masses in the adult patient: The role of percutaneous biopsy. Radiology 240:6-22

23. Uppot RN, Harisinghani MG, Gervais DA (2010) Imaging-guided percutaneous renal biopsy: rationale and approach. Ajr Am J Roentgenol 194:1443-1449

24. Veltri A, Bargellini I, Giorgi L et al (2017) CIRSE guidelines on percutaneous needle biopsy (PNB). Cardiovasc Intervent Radiol 40:1501-1513

25. Whittier WL, Korbet SM (2004) Timing of complications in percutaneous renal biopsy. J Am Soc Nephrol 15:142-147

26. Zagoria RJ (2000) Imaging of small renal masses: a medical success story. Ajr Am J Roentgenol 175:945-955

27. Zins M, Vilgrain V, Gayno S et al (1992) US-guided percutaneous liver biopsy with plugging of the needle track: a prospective study in 72 high-risk patients. Radiology 184:841-843 
Hier steht eine Anzeige.

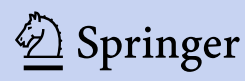

